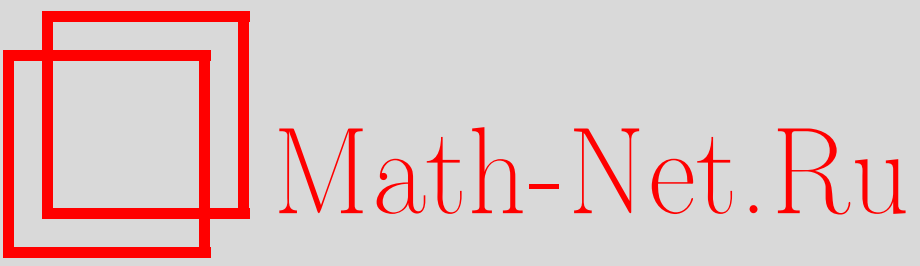

В. М. Овсянников, Уравнение неразрывности Эйлера с членами высокого порядка малости по времени течения, Итоги науки и техн. Сер. Соврем. мат. и ее прил. Темат. обз., 2020, том 182, 95-100

DOI: https://doi.org/10.36535/0233-6723-2020-182-95-100

Использование Общероссийского математического портала Math-Net.Ru подразумевает, что вы прочитали и согласны с пользовательским соглашением

http: //www.mathnet.ru/rus/agreement

Параметры загрузки:

IP : 54.80 .73 .141

26 апреля 2023 г., $16: 35: 49$ 


\title{
УРАВНЕНИЕ НЕРАЗРЫВНОСТИ ЭЙЛЕРА С ЧЛЕНАМИ ВЫСОКОГО ПОРЯДКА МАЛОСТИ ПО ВРЕМЕНИ ТЕЧЕНИЯ
}

\author{
(c) 2020 г. $\quad$ В. М. ОВСЯННИКОВ
}

\begin{abstract}
АннотАция. Рассматривается возникновение членов высокого порядка малости в уравнении неразрывности для несжимаемой жидкости, выведенном Эйлером в 1752 г. исходя из линейных по времени уравнений Коши-Гельмгольца. Согласно методу акустической аналогии Лайтхилла эти дополнительные члены проникают в неоднородную часть волнового уравнения и приводят к генерации автоколебаний и звуковых волн. В методе Лайтхилла волновое уравнение второго порядка по времени получается путем взятия производной по времени от уравнения неразрывности, при совершении которого члены второго порядка малости, которыми обычно пренебрегают, повышают свой порядок и становятся сопоставимыми с остальными членами волнового уравнения. Решение неоднородного волнового уравнения дает возможность понять причины возникновения, вычислить или оценить интенсивность вибраций и автоколебаний, которые в литературе рассматриваются иногда возникающими самопроизвольно.
\end{abstract}

Ключевые слова: уравнение неразрывности Эйлера, члены высокого порядка малости, формула Гаусса-Остроградского, формулы Коши-Гельмгольца, неоднородное волновое уравнение, генерация звука, автоколебания.

\section{EULER CONTINUITY EQUATION WITH HIGH-ORDER TERMS IN TIME}

\section{(c) 2020 V. M. OVSYANNIKOV}

\begin{abstract}
In this paper, we examine the appearance of high-order terms in the continuity equation for an incompressible fluid obtained by L. Euler in 1752 from the linear Cauchy-Helmholtz equations. According to Lighthill's acoustic analogy, these additional terms in the inhomogeneity of the wave equation lead to the generation of self-oscillations and sound waves. In Lighthill's method, the secondorder wave equation is obtained by taking the time derivative of the continuity equation. In this case, second-order terms that are usually neglected, increase their order and become comparable with other terms of the wave equation. Solution of the inhomogeneous wave equation allows one calculate or estimate the intensity of vibrations and self-oscillations, which are sometimes considered spontaneous.

Keywords and phrases: Euler continuity equation, high-order terms of smallness, GaussOstrogradsky formula, Cauchy-Helmholtz formulas, inhomogeneous wave equation, sound generation, self-oscillations.
\end{abstract}

AMS Subject Classification: 14C99, 76Q05

1. Дополнительные члены уравнения неразрывности Эйлера. Уравнение неразрывности, выражающее закон сохранения и являющееся основой гидрогазодинамики, имеет вид

$$
\operatorname{div} \boldsymbol{V}=0 \quad \text { или } \quad \frac{\partial u}{\partial x}+\frac{\partial v}{\partial y}+\frac{\partial w}{\partial z}=0,
$$

где $\boldsymbol{V}$ - вектор скорости с компонентами $u, v, w$ вдоль осей координат $x, y, z$. 
Оно было выведено геометрически в 1752 г. Леонардом Эйлером (см. [16]), а затем в 1830-е гг. было подтверждено с использованием формулы, названной позже формулой ГауссаОстроградского. Вывод Эйлера сводился к приравниванию объема недеформированного, начального тела объему тела, испытавшего три деформации растяжения или сжатия вдоль осей $x, y, z$, и три деформации сдвига вдоль каждой из этих осей $x, y, z$. Возникает вопрос, как шесть произвольных по величине деформаций могут быть учтены суммой трех слагаемых? На возможность существования в уравнении неразрывности членов высокого порядка обратил внимание В. А. Бубнов при изучении вывода уравнения неразрывности, сделанного Н. Е. Жуковским (см. $[1,2])$. Интуитивное чувство наличия дополнительного члена в уравнении неразрывности возникало и у авторов приема регуляризации уравнений гидрогазадинамики (см. $[3,10])$, добавлявших дополнительные члены при решении уравнений численными методами для улучшения сходимости итераций. Положение прояснил К. Трусделл, который в 1954 г. опубликовал краткий пересказ на английский язык с латыни вывода Эйлера (см. [17]), в котором оказалось, что шесть деформаций контрольной фигуры растяжения и сдвига контрольной фигуры вдоль трех осей координат приводят к возникновению 15 слагаемых уравнения неразрывности

$$
\frac{\partial u}{\partial x}+\frac{\partial v}{\partial y}+\frac{\partial w}{\partial z}+(\Delta t)\left[\frac{\partial(u, v)}{\partial(x, y)}+\frac{\partial(v, w)}{\partial(y, z)}+\frac{\partial(w, u)}{\partial(z, x)}\right]+(\Delta t)^{2} \frac{\partial(u, v, w)}{\partial(x, y, z)}=0 .
$$

Здесь $\Delta t$-интервал времени, за который происходит деформация исходной контрольной фигуры, через $\partial(u, v) / \partial(x, y)$ обозначены якобианы поля скорости второго порядка, $\partial(u, v, w) / \partial(x, y, z)$ - якобиан третьего порядка.

На следующем этапе вывода Эйлер предельными переходами, устремляя $\Delta t \rightarrow 0$, избавился от членов второго и третьего порядков малости, получив дифференциальное уравнение первого порядка $\operatorname{div} \boldsymbol{V}=0$. С позиций Лайтхилла, в 1952-1954 гг. предложившего вывод волнового уравнения методом акустической аналогии (см. $[18,19])$, содержащего взятие производной по времени $t$ от уравнения неразрывности, дополнительные члены могут попасть в неоднородную часть волнового уравнения и привести к генерации в потоке звуковых волн и автоколебаний.

В несжимаемой жидкости образование волн невозможно. Поэтому уравнение (1) в 2006 г. было переписано (см. [7]) для сжимаемого газа в виде

$$
\frac{\partial \rho}{\partial t}+\frac{\partial(\rho u)}{\partial x}+\frac{\partial(\rho v)}{\partial y}+\frac{\partial(\rho w)}{\partial z}+\rho(\Delta t)\left[\frac{\partial(u, v)}{\partial(x, y)}+\frac{\partial(v, w)}{\partial(y, z)}+\frac{\partial(w, u)}{\partial(z, x)}\right]+\rho(\Delta t)^{2} \frac{\partial(u, v, w)}{\partial(x, y, z)}=0,
$$

где $\rho$ - плотность газа.

Отметим, что учет сжимаемости и переменности плотности в общепринятом дифференциальном уравнении неразрывности

$$
\frac{\partial \rho}{\partial t}+\frac{\partial(\rho u)}{\partial x}+\frac{\partial(\rho v)}{\partial y}+\frac{\partial(\rho w)}{\partial z}=0
$$

не дает возникновения волн. Сжимаемость приводит только к возможности пропуска через газ волн, уже созданных граничными условиями за счет подвижных твердых стенок или другими средствами. Возникновение новых волн и усиление уже существующих дается дополнительными членами уравнения неразрывности, вычисленными Эйлером.

Следует напомнить, что существует еще другой механизм генерации волн за счет конвективных членов уравнения неразрывности, найденный Л. Д. Ландау и Е. М. Лифшицем (см. [5, § 75]).

2. Физический смысл членов второго порядка уравнения неразрывности - генерация периодических волн. Возвращаясь к уравнению неразрывности (2), учитывающему и сжимаемость и выведенные Эйлером члены второго порядка малости с якобианами второго порядка, отметим, что метод акустической аналогии Лайтхилла с его использованием дает неоднородное волновое уравнение (см. $[8,9])$.

$$
c_{0}^{-2} \frac{\partial^{2} p}{\partial t^{2}}-\frac{\partial p^{2}}{\partial x^{2}}-\frac{\partial^{2} p}{\partial y^{2}}-\frac{\partial^{2} p}{\partial z^{2}}=-\rho_{0} J
$$


где

$$
J=\frac{\partial(u, v)}{\partial(x, y)}+\frac{\partial(v, w)}{\partial(y, z)}+\frac{\partial(w, u)}{\partial(z, x)}
$$

- сумма якобианов второго порядка вектора скорости, $\rho_{0}-$ средняя плотность, $c_{0}-$ скорость звука. Это уравнение было выведено в 2007 г. (см. [8, с. 22]).

Появление нового механизма расчета образования волн из членов второго порядка малости уравнения неразрывности, которые всегда опускали, ставит два вопроса. Первый вопрос: не будет ли эффект волнообразования таким слабым, что его практический вклад в образование звуковых волн и автоколебаний будет очень мал? Для ответа на него был просчитан комплекс инженерных и естественнонаучных задач (см. [13,15]), который показал значительность интенсивности возникающих колебаний и возможность накопления волновой энергии в течение длительного времени в упругих конструкциях. Речь шла об инженерных сооружениях с размерами, меняющимися от метра до километра. Расчет естественнонаучных задач охватывал широкий круг тем от сейсмических задач по течению магмы до задачи колебаний атмосферы Солнца.

Второй вопрос состоял в отсутствии дополнительных членов высокого порядка малости в другом, широко распространенном выводе уравнения неразрывности, проводящимся с использованием формулы Гаусса-Остроградского.

3. Проявление членов второго порядка малости в уравнении неразрывности, выводящемся с использованием формулы Гаусса-Остроградского. Для обоснованного и уверенного использования уравнения неразрывности Эйлера с членами второго порядка малости по времени движения необходимо найти члены второго порядка малости в выводе уравнения неразрывности, проводящимся с использованием формулы Гаусса-Остроградского:

$$
\iiint_{(V)}\left(\frac{\partial P}{\partial x}+\frac{\partial Q}{\partial y}+\frac{\partial R}{\partial z}\right) d x d y d z=\iint_{(S)}(P \cos \lambda+Q \cos \mu+R \cos \nu) d S .
$$

Обратим внимание, что построения Эйлера, дающие члены второго порядка малости, проведены геометрически на базе элементарной геометрии без использования предельных переходов устремления к нулю ни интервалов времени $\Delta t$, ни размеров $\Delta x, \Delta y, \Delta z$. Поэтому в формуле Гаусса-Остроградского можно увидеть члены второго порядка малости $\varepsilon$, содержащие время движения жидкой частицы, если заменить интегралы интегральными суммами

$$
\sum \sum_{(V)} \sum\left(\frac{\partial P}{\partial x}+\frac{\partial Q}{\partial y}+\frac{\partial R}{\partial z}\right) \Delta x \Delta y \Delta z=\varepsilon+\sum_{(S)}(P \cos \lambda+Q \cos \mu+R \cos \nu) \Delta S
$$

и разобрать кинематику перемещения жидких частиц, пересекающих границу контрольной фигуры из окружающего пространства. Напомним, что интеграл можно вводить и рассматривать как предел интегральной суммы при устремлении к нулю $\Delta x, \Delta y, \Delta z$.

Анализ перемещений жидких частиц в 2017 г. был проведен для потенциального течения жидкости или газа внутри прямого угла и опубликован в [11, с. 152-155, 208-209, 244-246].

Эффект шагреневой кожи уменьшения площади от времени в формуле Гаусса-Остроградского при потенциальном течении внутри прямого угла с

$$
\frac{\partial u}{\partial y}=0, \quad \frac{\partial v}{\partial x}=0
$$

иллюстрируют рис. 1 и рис. 2.

Невязкая жидкость обтекает прямой угол по траекториям, имеющим форму гипербол. Использование формулы Гаусса-Остроградского с направляющими косинусами заменяет вблизи поверхности квадратной контрольной фигуры действительные гиперболические траектории на прямолинейные (см. рис. 1). При использовании искажения поля скорости обтекания прямого угла направляющими косинусами в контрольную фигуру втекает столько же жидких частиц, сколько вытекает, и выполняется точно закон сохранения. При учете точного поля скорости без использования направляющих косинусов (см. рис. 2) баланс числа частиц нарушается и втекает в выпуклую контрольную фигуру в единицу времени меньше жидких частиц, чем вытекает из 


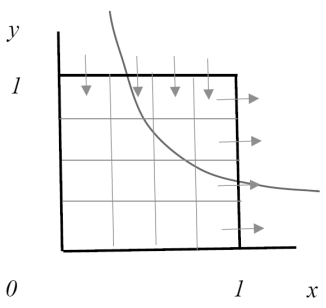

Рис. 1

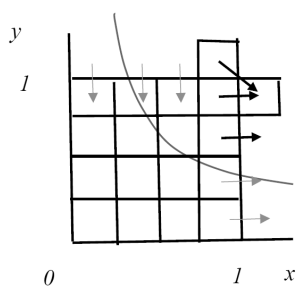

Рис. 2

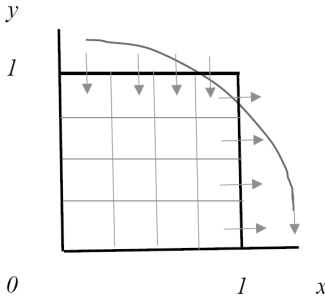

Рис. 3

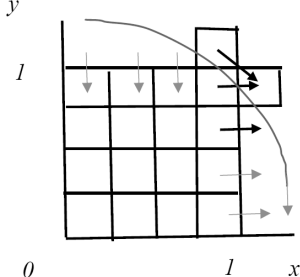

Рис. 4

нее. Арифметический расчет (см. [11, с. 152-155, 208-209, 244-246]) дает точно такую же величину нарушения баланса количества вещества, как и члены второго порядка малости уравнения неразрывности Эйлера.

Сейчас это можно продемонстрировать для течения, содержащего деформации сдвига при вращательном движении жидкости с

$$
\frac{\partial u}{\partial y} \neq 0, \quad \frac{\partial v}{\partial x} \neq 0
$$

на рис. 3 и 4.

На рис. 3 показаны направления перемещения жидких частиц при использовании направляющих косинусов. Баланс количества вещества выполняется точно. На рис. 4 указаны действительные направления перемещения жидких частиц без искажения направляющими косинусами. В угловой точке, самой удаленной от центра вращения, происходит такая же кинематическая картина покидания веществом контрольной фигуры и повышенное сосредоточение его в соседнем объеме такого же размера. Арифметический расчет дает точно такое же нарушение баланса, как и члены второго порядка малости уравнения неразрывности Эйлера. Это локальное несохранение дает кинематическую картину образования волны уплотнения вещества.

Заменив математическое понятие потока произведением скорости на площадь поверхности (или длину границы в плоском двухмерном течении) контрольной фигуры и не используя направляющих косинусов, мы получаем, что часть жидких частиц дважды пересечет по секущей границу контрольной фигуры за интервал времени $\Delta t$, выйдет за пределы контрольной фигуры и не будет учтена в балансе вещества.

Оба примера расчета показывают, что члены высокого порядка малости, отражающие локальное несохранение, совпадают с дополнительными членами, вычисленными Эйлером. Примеры охватывают потенциальные течения и течения с деформациями сдвига. Таким образом, мы проиллюстрировали, что и вывод Эйлера уравнения неразрывности и вывод с использованием теоремы Гаусса-Остроградского дают уравнение неразрывности, содержащее члены высокого порядка малости по времени течения и дают волновое уравнение, генерирующее звук и автоколебания.

4. Линейность лагранжева закона движения - причина возникновения звуковых волн и автоколебаний. Причиной возникновения членов высокого порядка малости и у Эйлера и в подходе вывода формулы Гаусса-Остроградского является использование линейных по времени формул Коши-Гельмгольца

$$
x=(1+a t) x_{b}+b t y_{b}, \quad y=c t x_{b}+(1+k t) y_{b},
$$

где $x_{b}, y_{b}$ - начальные координаты движущейся жидкой точки; $t$ - время движения; $a, b, c, k-$ коэффициенты, характеризующие поле скорости, в качестве лагранжева закона движения жидкой частицы. В самом деле, при вычислении объема как произведения трех линейных функций, будет возникать кубический по времени член в балансе вещества для пространственного трехмерного течения. Произведение двух линейных функций в плоском течении будет давать квадратичный член в балансе вещества. 
При сложном экспоненциальном лагранжевом законе движения жидкой частицы (см. [11, (3.5), c. 52]) будет выполняться точное сохранение количества вещества во времени. Но, как показано в [11], экспоненциальный лагранжев закон движения жидкой частицы соответствует полю ускоренного течения. Этот расчет подтверждают гидрогазодинамические эксперименты. Ускоренные течения проявляют исключительную способность двигаться в ламинарном режиме, не создавая турбулентных пульсаций. Для них переход к турбулентному течению происходит при числах Рейнольдса Re, в сотни раз превышающих критические значения Re для стационарных течений (см. $[4,6])$.

Но все поле течения не может состоять только из участков ускоренного течения. Имеются и замедленные течения, и течения с небольшими ускорениями. В этих областях и зарождаются автоколебания и звуковые волны, интенсивность которых можно вычислить или оценить по приведенной выше модели Эйлера и обсуждаемой ниже модели Ландау-Лифшица.

5. Образование волн за счет учета конвективных членов уравнения движения. Лайтхилл (см. $[18,19])$ в 1952-54 гг. предложил формальный вывод волнового уравнения из системы уравнений газовой динамики. Он линеаризовал уравнения неразрывности для нестационарного течения сжимаемого газа и уравнение движения. Затем он взял производную от уравнения неразрывности по времени, а от уравнения движения - по координате и вычел один результат из другого. В результате получается однородное волновое уравнение - «уравнение струны». Если же в исходные уравнения добавить члены турбулентности, то они проникают в неоднородную часть волнового уравнения и дают источник генерации колебаний и турбулентных пульсаций. Это сделал Лайтхилл.

Л. Д. Ландау и Е. М. Лифшиц (см. [5]) повторили линеаризацию уравнений, но в уравнении движения оставили конвективные члены, которые у Лайтхилла при линеаризации исчезли. В результате Ландау и Лифшиц получили неоднородное волновое уравнение, приведенное в [5, § 75]. Вычисление (см. [14]) неоднородного члена, проведенное в 2017 г., дало волновое уравнение вида (3), в котором неоднородная часть имела такую же структуру, но была в 2 раза больше:

$$
c_{0}^{-2} \frac{\partial^{2} p}{\partial t^{2}}-\frac{\partial p^{2}}{\partial x^{2}}-\frac{\partial^{2} p}{\partial y^{2}}-\frac{\partial^{2} p}{\partial z^{2}}=-2 \rho_{0} J
$$

Все инженерные примеры и оценки для естественнонаучных явлений, указанные в разделе 2 , были проведены с учетом суммы воздействий на генерацию периодических волн и конвективных членов уравнения движения и членов высокого порядка уравнения неразрывности Эйлера.

$$
c_{0}^{-2} \frac{\partial^{2} p}{\partial t^{2}}-\frac{\partial p^{2}}{\partial x^{2}}-\frac{\partial^{2} p}{\partial y^{2}}-\frac{\partial^{2} p}{\partial z^{2}}=-3 \rho_{0} J
$$

В связи с появлением в уравнении неразрывности - законе сохранения дополнительных членов второго порядка малости - возник вопрос о совместимости получающегося локального несохранения Эйлера (см. [12]) с интегральным законом сохранения Древних, который отстаивал М. В. Ломоносов. Надо обратить внимание на то, что и у философов античного мира, и у М. В. Ломоносова постулировалось сохранение вещества или количества атомов в одном выделенном и изолированном от окружающего объеме. Дифференциальный и локальный подход предполагает наличие множества или хотя бы нескольких малых соседних смежных объемов - 4 или 8 в плоском течении и 6 или 14 в пространственном трехмерном. С соседними объемами может происходить обмен веществом или частичный переток без его компенсации. Звуковая волна плотности как раз и означает периодическое возрастание и убывание вещества в контрольной фигуре. Поэтому дополнительные члены второго порядка малости, линейные по времени в уравнении неразрывности, приводят к возникновению периодических гармонических колебаний и не нарушают общего интегрального баланса вещества в закрытом помещении. Напомним, что одной переменности плотности достаточно, чтобы передавался через газ звук, генерируемый подвижной стенкой. Но чтобы звук возникал в газе без участия граничных условий, надо иметь в системе уравнений конвективные члены уравнения движения или члены высокого порядка малости уравнения неразрывности Эйлера. Периодический, гармонический характер автоколебаний позволяет совместить 
интегральное сохранение с локальным дифференциальным несохранением с гармоническими зависимостями от времени и от координаты.

\section{СПИСОК ЛИТЕРАТУРЫ}

1. Бубнов B. A. Физические принципы гидродинамических движений// в кн.: Проблемы аксиоматики в гидрогазодинамике. - М.: Прометей, 1997. - Т. 4. - С. 206-269.

2. Бубнов В. И. Кинематика жидкой частицы// в кн.: Проблемы аксиоматики в гидрогазодинамике. М.: Прометей, 1999. - Т. 7. - С. 11-29.

3. Елизарова Т. Г. Квазигазодинамические уравнения и методы расчета вязких течений. - М.: Научный Мир, 2007.

4. Коппель Т. А., Лийв У. Р. Экспериментальное исследование возникновения движения жидкости в трубопроводах // Изв. АН СССР. Мех. жидк. газа. - 1977. - № 6. - С. 78-85.

5. Ландау Л. Д., Лифиии, Е. М. Гидродинамика. - М.: Наука, 1986.

6. Овсянников В. М. Расчет возникновения движения жидкости в трубопроводе// Изв. АН СССР. Мех. жидк. газа. - 1981. - № 5. - С. 158-160.

7. Овсянников В. М. Введение в аксиоматическую механику жидкости, основанную на базисных экспериментах с жидкостью// в кн.: Проблемы аксиоматики в гидрогазодинамике. - М., 2006. - Т. 15. C. $19-51$.

8. Овсянников B. M. Разностная форма уравнения неразрывности с учетом деформаций сдвига// в кн.: Проблемы аксиоматики в гидрогазодинамике. - М., 2007. - Т. 16.

9. Овсянников В. М. История вывода уравнения неразрывности// Мат. XI Всеросс. съезда по фундам. пробл. теор. и прикл. мех. (Казань, 20-24 августа 2015 г.). - Казань, 2015. - С. 2823-2824.

10. Овсянников В. М. Сопоставление дополнительных слагаемых второго порядка малости для конечноразностных уравнений Эйлера и малых добавок в регуляризованных уравнениях гидродинамики// Ж. вычисл. мат. мат. физ. - 2017. - № 5. - С. 876-880.

11. Овсянников В. М. Волнообразование и конечно-разностное уравнение неразрывности Леонарда Эйлера. - М.: Спутник+, 2017.

12. Овсянников В. М. Локальное дифференциальное несохранение при интегральном сохранении в газовой динамике. - М.: Спутник,+ 2017.

13. Овсянников В. М. Эллипсоид деформации Н. Е. Жуковского с учетом членов второго порядка малости// Инж. ж. Наука иннов. - 2018. - № 5 (77). - С. 1-11.

14. Овсянников B. М. Вибратор Ландау-Лифшица в уравнениях газовой динамики// Инж. ж. Наука иннов. - 2018. - № 4 (76). - С. 1-8.

15. Овсянников В. М. Использование аксиомы Лейбница теории бесконечно малых и аксиомы Ньютона теории исчезающе малых величин при выводе волнового дифференциального уравнения// Тез. докл. Междунар. конф. по дифференциальным уравнениям и динамическим системам (Суздаль, 6-11 июля 2018 г.). - Суздаль, 2018. - С. 155-156.

16. Эйлер Л. Общие законы движения жидкостей// Мех. жидк. газа. - 1999. - 6. - С. $26-54$.

17. Euler L. Commentationes Mechanicae ad Theoriam Corporum Fluidorum Pertinentes. — Zürich: Orell Fuessli, 1954.

18. Lighthill M. J. On sound generated aerodynamically. I. General theory// Proc. Roy. Soc. Lond. Ser. A 1952. - 211. - P. 564-587.

19. Lighthill M. J. On sound generated aerodynamically. I. Turbulence a source of sound// Proc. Roy. Soc. Lond. Ser. A - 1954. - 222. - P. 1-32.

Овсянников Владислав Михайлович

Московская государственная академия водного транспорта;

Ноябрьский институт нефти и газа - филиал Тюменского индустриального университета

E-mail: OvsyannikovVM@yandex.ru 